ARCHIVOS

de historia del movimiento obrero y la izquierda
ISSN 2313-9749

ISSN en línea 2683-9601

Año VIII, $\mathrm{n}^{\circ} 16$, pp. 17-36

marzo de 2020-agosto de 2020

\title{
Las visiones del trabajo en la teoría feminista
}

\author{
Susan Ferguson \\ Universidad Wilfrid Laurier (Waterloo, Canadá)·sferguson@wlu.ca
}

Titulo: Visions of Work in Feminist Theory

Resumen: Este artículo recorre las diversas formas en que el trabajo ha sido objeto de análisis en la teoría feminista. Identifica dos enfoques analíticos generales que, si bien comparten la idea de que la devaluación social del trabajo que realizan las mujeres es una característica definitoria de la desigualdad y la opresión que sufren, difieren en sus conceptualizaciones sobre el poder social y, por ende, en sus proyectos políticos para la emancipación de las mujeres. E1 primero, "feminismo de la igualdad", se centra en la división sexual del trabajo; el segundo, "feminismo de la reproducción social", se central en el trabajo reproductivo como elemento clave para la reproducción continua de la sociedad capitalista. El artículo analiza ambas perspectivas y da los argumentos de por qué el segundo enfoque logra superar las debilidades del primero.

Palabras clave: trabajo - capitalismo - feminismo de la igualdad - feminismo de la reproducción social

\begin{abstract}
This article takes a look at the various ways in which labor has been assessed in feminist theory, within which two broad analytic approaches can be discerned. They both identify the societal devaluing of and restrictions on the work women do as defining features of women's inequality and oppression, but they differ in their conceptualizations of social power and, relatedly, in their political projects for women's emancipation. The first one, "equality feminism", focuses on the sexual division of labor, whereas the second one, "social reproduction feminism", focuses on reproductive labor as a key element in the ongoing reproduction of capitalist society. The article analyses both perspectives and argues that the second approach succeeds in overcoming the weaknesses of the first.
\end{abstract}

Keywords: labor - capitalism - equality feminism - social reproduction feminism

Recepción: 16 de diciembre de 2019. Aceptación: 27 de febrero de 2020 


\section{Introducción ${ }^{1}$}

El trabajo ha sido una preocupación para la teoría feminista desde hace mucho tiempo. Ya en 1792, en su Vindicación de los derechos de la mujer, Mary Wollstonecraft destaca el valor del trabajo de las madres y las esposas, y afirma que una educación igualitaria para las mujeres beneficiaría su trabajo como médicas, comerciantes y en otras profesiones. Desde entonces, las feministas han retomado el tema, a menudo avanzando hacia una comprensión más crítica, matizada y compleja de qué constituye trabajo, quién trabaja, bajo qué condiciones y cómo se mide el valor del trabajo. Y durante más de doscientos años han propuesto estrategias para la emancipación de las mujeres basadas en nuevas formas de imaginar su trabajo.

Dentro de esta rica y variable historia del pensamiento feminista sobre el trabajo, es posible distinguir dos enfoques analíticos generales. Si bien ambos identifican la devaluación social del trabajo que realizan las mujeres y las restricciones al mismo como características definitorias de la desigualdad y de la opresión de las mujeres, difieren en sus conceptualizaciones sobre el poder social y, en consecuencia, en sus proyectos políticos para la emancipación de las mujeres. El primero se centra en la división sexual del trabajo, la cual refiere a las convenciones sociales que asignan a las mujeres la responsabilidad de la reproducción fisiológica, la crianza de los hijos y el "trabajo doméstico" en las diferentes sociedades de clase, al tiempo que asignan a los hombres otras tareas "productivas". Esta línea crítica condena las desventajas que este "destino" implica para las mujeres, y lo injusto de las ventajas sociales y económicas de los hombres. Se examinan críticamente la naturaleza del trabajo de las mujeres y las condiciones bajo las cuales lo realizan, en contraste con la naturaleza y las condiciones del trabajo que realizan los hombres. La división sexual del trabajo es generalmente (aunque no siempre) rechazada como algo antinatural o injusto. Y, debido a que se supone que la libertad reside en la independencia económica de las mujeres, la principal estrategia política es la integración de las mujeres en la fuerza de trabajo remunerada en pie de igualdad con los hombres.

El segundo enfoque analítico se centra en las formas bajo las cuales el control patriarcal del trabajo de las mujeres está relacionado con la reproducción continua de la sociedad capitalista. No es la división sexual

1. La versión en inglés de este artículo forma parte de The Bloomsbury Handbook of 21st-Century Feminist Theory editado por Robin Truth Goodman y publicado en 2019 por la editorial Bloomsbury del Reino Unido. Agradecemos a Susan Ferguson y a la editorial Bloomsbury por haber autorizado su publicación en español para este número de la revista Archivos. La traducción estuvo a cargo de Antonio Oliva y Paula Varela. 
del trabajo en sí misma la que explica la opresión de las mujeres, sino el hecho de que esta división está basada, de manera contradictoria y parcial, en la lógica esencialmente deshumanizante de la acumulación capitalista. El trabajo, en este caso, se concibe en un sentido amplio, y se destaca el valor del trabajo doméstico de las mujeres. Sin embargo, ese valor no solo se evalúa en una escala moral (por su mérito para garantizar una sociedad buena y justa). Se lo analiza en términos político-económicos (por su capacidad para asegurar la continuidad de la sociedad capitalista). La libertad es imaginada en este caso como una reorganización general de todo el trabajo con el fin de interrumpir la tendencia del capitalismo a privatizar y deshumanizar los procesos de producción involucrados en la satisfacción de las necesidades de subsistencia.

Este artículo revisa las trayectorias históricas de estos dos análisis, en lo sucesivo denominados "feminismo de la igualdad" y "feminismo de la reproducción social". Se remonta, de modo necesariamente somero, a lo largo de los últimos dos siglos para destacar sus interpretaciones, relacionadas aunque diferenciadas, de la importancia del trabajo en el pensamiento feminista. El artículo también critica el individualismo y las limitaciones de clase de la lente con la cual el feminismo de la igualdad ve el mundo del trabajo de las mujeres; limitaciones que, a mi juicio, logra superar el enfoque feminista de la reproducción social.

\section{El feminismo originario: el potencial igualador del trabajo}

Lo que las mujeres hacen -y lo que se les ha impedido hacer- constituye gran parte de su experiencia real de opresión. Por lo tanto, no es sorprendente que el "trabajo" aparezca como una preocupación destacada en las primeras teorías feministas. Escribiendo al comienzo de la primera revolución industrial, muchas feministas aceptaron la división del trabajo por género que consideraba a las mujeres naturalmente aptas para dirigir los hogares y criar a los hijos. Sin embargo, pidieron reformas para abordar la relación desigual de mujeres y hombres con el trabajo. En Vindicación.., por ejemplo, Wollstonecraft argumenta que permitir a las mujeres de "sectores medios" y de "la clase superior" acceder a la educación de los clásicos, probaría y mejoraría la capacidad de las mujeres para aplicar principios racionales y morales a sus deberes domésticos (lo que ella ve como su vocación natural), así como a sus ocupaciones laborales (menciona la medicina, la enfermeria, la partería y la administración de una tienda o una granja) (1999, cap. 4). Otras demócratas radicales de la década de 1790 se aventuraron a criticar más directamente la posición de las mujeres en el mercado laboral. Olympe de Gouges aboga por que los hombres y las mujeres compar- 
tan igualitariamente todos los "puestos, empleos, oficinas, honores y trabajos" (Gouges, 2017, art. XIII), mientras que Mary Anne Radcliffe refiere a la usurpación del trabajo asalariado por los hombres como "la gran causa" de la pobreza de las mujeres y su consecuente vuelco al robo y la prostitución $(1799$, p. 86). En lugar de ver con malos ojos a aquellas que no tienen más remedio que "buscar el pan en los caminos del vicio", insta a que la sociedad ofrezca caridad y trabajo $(1799$, p. 46).

En todos estos análisis, el trabajo en sí mismo es visto como algo positivo. Se propone un trabajo útil y racional como correctivo de la degradación y la desigualdad de las mujeres, así como de la corrupción de su carácter provocado por el maltrato que les propina la sociedad. De hecho, Wollstonecraft y Radcliffe sugieren que las mujeres más ricas tienen algo que aprender de la "laboriosidad" de sus hermanas trabajadoras más pobres. Ninguna de las dos considera que la naturaleza servil y las penurias del trabajo doméstico u otro trabajo de servidumbre sean un problema del feminismo. Wollstonecraft deja en claro este punto en su descripción de las relaciones libres e iguales entre los sexos, afirmando que la esposa depende "simplemente de una sirvienta para dejar de hacerse cargo de la parte servil de las tareas del hogar" (1999, cap. 9). La igualdad aparece como algo a ser conquistado sobre la condición de que haya una mujer trabajadora pobre que esté disponible para fregar los pisos y cambiar los pañales.

En otras palabras, las primeras observaciones feministas acerca de la relación de las mujeres con el trabajo descansan en una naturalización de las relaciones de clase capitalistas. ${ }^{2}$ Este es un tema que atravesó el análisis feminista de la igualdad durante los siguientes doscientos años. Otros dos temas -más progresivos- también están presentes en estas primeras consideraciones. Primero, aunque son relativamente indiferentes al trabajo doméstico, estas feministas ofrecen una visión crítica de la división sexual del trabajo de la fuerza laboral remunerada que, en el caso de Radcliffe, supone un desafio explícito a la naturalización de los roles de género, que prohíbe a las mujeres ejercer ciertas ocupaciones. En segundo lugar, Radcliffe ubica explícitamente la independencia financiera como un objetivo feminista. El camino hacia la igualdad y la libertad, sugiere, radica en mejorar el acceso de las mujeres al trabajo remunerado. Este énfasis en el potencial igualador del trabajo fue acogido, como veremos, no solo por las feministas liberales posteriores, sino también por muchas feministas socialistas.

2. Las demócratas radicales de 1790 , en general, criticaron la desigualdad, no el capitalismo y la dinámica de clase. 


\section{El problema del trabajo doméstico}

Un enfoque más crítico para teorizar el trabajo encuentra sus raíces en el siglo XIX, cuando las feministas comenzaron a prestar más atención a la crianza de los hijos y al mantenimiento del hogar. En lugar de una cuestión del deber de las mujeres, propusieron que tales actividades no eran inherentemente edificantes, aun cuando fueran socialmente valiosas. Sus análisis dieron lugar a algunas propuestas sorprendentemente modernas para aliviar las cargas del trabajo doméstico y redefinir el significado de la independencia de las mujeres. A su vez, abrieron el camino a la crítica de las características de clase del trabajo de las mujeres.

Ese camino se vislumbró más abiertamente en la década de 1820 en Gran Bretaña. En su tratado de 1825, los socialistas utópicos William Thompson y Anna Wheeler describieron la esclavitud doméstica de las mujeres con palabras exhortativas, refiriéndose al hogar como "la eterna prisión de la esposa" (1825, p. 79). ${ }^{3}$ Pero su pensamiento va más allá: de una condena moral se pasa a una crítica político-económica. La clave que aportaron, aun cuando no desarrollaron completamente la idea, es que el capitalismo no se sustenta simplemente en la explotación del trabajo asalariado; también depende en forma sustancial de la privatización del trabajo doméstico (de las mujeres) y, por lo tanto, de la opresión de las mismas. Es decir, el sistema económico es, en el fondo, también un sistema de opresión de género precisamente porque el trabajo doméstico es, en el capitalismo, un asunto privado.

Aunque esta idea inspiró experimentos de vida comunitaria durante muchos años, se mantuvo sin desarrollar como teoría de la opresión de las mujeres. ${ }^{4}$ En su libro El origen de la familia, la propiedad privada y el estado, de 1884, Friedrich Engels explica que la transición histórica de la propiedad comunitaria a la propiedad privada concentró la riqueza en manos de los hombres, lo que hizo que tomaran "el mando en el hogar", mientras que las mujeres fueron "degradadas y reducidas a servidumbre" (1972, pp. 120-121). Pero, debido a que los trabajadores no poseen propiedades bajo el capitalismo, razona Engels, el patriarcado carece de base material dentro de su clase. En su opinión (y en la de Marx), las mujeres deberian unirse a la fuerza de trabajo asalariada para obtener la independencia necesaria en función de enfrentar cualquier vestigio de patriarcado que persista en sus hogares. Como trabajadoras asalariadas, podrian participar en la lucha colectiva para derrocar el capitalismo (y

3. El exordio lleva el nombre de Thompson, aunque él atribuye las ideas a Wheeler.

4. Para las discusiones sobre los experimentos de socialización del trabajo doméstico en los Estados Unidos ver Hayden (1982) y también Siegel (1993-1994). 
con ello, la base material -la propiedad privada- de la opresión de las mujeres). ${ }^{5}$ En lugar de desarrollar la idea de que el trabajo doméstico de las mujeres es una piedra angular en el edificio capitalista, Engels propuso una variante de la teoría del potencial igualador que el trabajo remunerado tiene para las mujeres.

La posición de Engels fue predominante en la izquierda marxista durante el siglo siguiente: la cuestión del trabajo doméstico surgió solo de manera esporádica. Dos historias clásicas del trabajo de las mujeres revelan dos perspectivas divergentes: Working Life of Women in the Seventeenth Century de Alice Clark, editada en 1919 y Women Workers and the Industrial Revolution 1750-1850 de Ivy Pinchbeck, publicada once años después. Al rastrear el cambio del hogar rural al trabajo por piezas y la producción industrial, Pinchbeck presenta una imagen triunfalista en la que las penurias del trabajo remunerado de las mujeres disminuyen gradualmente a medida que la tecnologia, las concesiones capitalistas al movimiento obrero y las regulaciones estatales se coaligan para mejorar su situación. Al término de la revolución industrial, insiste, las mujeres solteras podían contar con su independencia financiera, y "la emancipación de las mujeres trabajadoras definitivamente había comenzado" (Pinchbeck, 1985, p. 314). Clark, por otro lado, es mucho menos optimista. La industrialización capitalista, argumenta, socava la "capacidad productiva" de las mujeres (1992, p. 290) haciendo la vida mucho más dificil para las mujeres de la clase trabajadora, en parte porque se concentraban en los trabajos peor pagos. Además de ser una de las primeras en señalar claramente la guetización del trabajo asalariado de las mujeres, plantea la cuestión de la relación entre el trabajo remunerado y no remunerado, sosteniendo que este último es tan productivo como el remunerado.

Mientras que gran parte de la izquierda socialista organizada tendía a evitar las discusiones sobre el trabajo doméstico, considerándolas irrelevantes para su proyecto político, las feministas liberales las incorporaron. Sin embargo, en lugar de preguntar de qué manera cocinar y limpiar sostiene el capitalismo, adoptaron y ampliaron una crítica moralista e individualista que condujo a estrategias para liberar a las mujeres del trabajo doméstico. Sus numerosas propuestas van desde reclamar que los hombres compartan "las cargas domésticas comunes" (Brown Blackwell, 1975, p. 155) hasta imaginar avances tecnológicos vinculados con la limpieza y la eliminación de desechos. Otras, perspicazmente, trazaron una línea directa entre trabajo remunerado y no remunerado,

5. Engels consideró que el fin del capitalismo también implicaba la socialización de la limpieza, el cuidado de los niños y la educación. Pero en ningún momento sugirió que estas tareas fueran parte integral de la reproducción en curso del capitalismo. 
y plantearon que las normas y reglamentos que se aplicaban en las industrias también fueran puestos en práctica en los hogares. En lugar de esperar que los hombres hagan el trabajo de las mujeres, Charlotte Perkins Gilman sugiere que el trabajo de "calefacción, iluminación, alimentación, vestimenta y limpieza" debe organizarse en una "amplia combinación de actividades bien administrada. . . [que asegure] horas regulares de trabajo y tiempo libre de descanso". También reclama "una buena remuneración para cada tipo de servicio" (1975, pp. 177-178). Décadas más tarde, Edith Stern extiende esta línea de razonamiento. La ama de casa es la "trabajadora olvidada" que no goza de salario mínimo, no tiene normas de higiene y seguridad y carece de periodos obligatorios de descanso. "Las personas libres, en una democracia, prestan servicios personales para sí mismas o, si tienen efectivo, pagan a otros individuos libres para que los atiendan" (Stern, 1975, p. 353).

Estas feministas creían que la libertad de las mujeres podía asegurarse mejorando el sistema competitivo, y no alterándolo. Los bienes y servicios producidos en forma capitalista, así como la absorción del trabajo doméstico en las relaciones de producción capitalistas reguladas, liberarian a las mujeres de la esclavitud del hogar. Si bien sus críticas resaltan el valor social del trabajo doméstico y de crianza no remunerado, nunca se cuestionan por qué existe la evidente división entre el trabajo remunerado y no remunerado. Tampoco reconocen las experiencias opresivas específicas de los sectores obreros que producen los bienes y servicios que, supuestamente, emanciparian a las mujeres: sectores que, en su mayoria, también están compuestos por mujeres. En su lugar, el trabajo asalariado y el "individuo libre" de Stern, que es capaz o bien de pagarlo o bien de realizarlo, son considerados como el modelo y el medio para alcanzar la independencia y la libertad.

La insuficientemente teorizada relación entre trabajo remunerado y no remunerado siguió estando presente en la agenda feminista en la era posterior a la Segunda Guerra Mundial. Un crecimiento sostenido en la productividad y la ampliación de la provisión en materia de seguridad social llevaron a muchos trabajadores, aunque no a todos, a disfrutar de salarios más altos y un mejor nivel de vida. Este relativo bienestar, que fue disfrutado desproporcionadamente por los hogares blancos y encabezados por varones en América del Norte y Europa Occidental, permitió a ciertas mujeres de la clase trabajadora optar por no trabajar en forma remunerada. ${ }^{6}$ Dedicarse a tiempo completo a ser madres y

6. Los afroamericanos y otros inmigrantes no solo fueron parte de manera desigual del bienestar de la época, sino que los criterios de elegibilidad racistas para medidas de asistencia pública como la Ayuda para niños dependientes en los Estados Unidos (más tarde conocida como Ayuda para familias con niños dependientes) también 
"amas de casa" (de manera no remunerada), se convirtió en el modelo de feminidad para un número cada vez mayor de mujeres. Sin embargo, mientras en muchas amas de casa resonaba la idea de que la opresión de las mujeres se basaba en la monotonía del trabajo doméstico y sus efectos aislantes, era esta misma idea la que eclipsaba las experiencias de millones de mujeres inmigrantes y negras. Estas eran las mujeres que fregaban los pisos y limpiaban las narices de los niños en hogares que no eran los suyos, en hogares blancos, por salarios extremadamente bajos y con escaso control sobre las condiciones de su trabajo. Como escribe elocuentemente Angela Davis sobre las mujeres afroamericanas: "Aunque rara vez han sido "solo amas de casa", siempre han hecho sus tareas domésticas" (1982, p. 231).

En ese sentido, Claudia Jones señala en 1949 que, además de ocuparse de sus propios hogares, las mujeres negras en los Estados Unidos tenían el doble de probabilidades de tener que trabajar para ganarse la vida y hacerlo por menos de la mitad del salario que las mujeres blancas. Para estas mujeres el trabajo doméstico no era, por lo tanto, un marcador del estatus de clase media ni una experiencia aislante. Por el contrario, el trabajo doméstico remunerado indicaba pobreza $y$ racismo. Y este es el punto central de Jones. Denunciando la "súper explotación" y la "degradación" de las mujeres negras, Jones presagia las teorizaciones del feminismo negro sobre opresiones "intersectadas", insistiendo en que la "mujer negra . . . [enfrenta] una opresión especial como negra, como mujer y como trabajadora" (1949, p. 7). Más aún, el trabajo doméstico no remunerado y la crianza de los hijos de las mujeres negras tienen su propia historia y significado diferenciados.

Jones introdujo una notable complejidad en la comprensión feminista del trabajo de las mujeres y de su poder social. Mientras que (al igual que sus camaradas del Partido Comunista), Jones vio la clave para la emancipación de las mujeres (negras) en la lucha por el mejoramiento de sus salarios y condiciones, fue un paso más allá al argumentar que el trabajo no es solo un problema "económico". Insistió en que, debido a que la fuerza de trabajo estadounidense estaba tan racializada, no podría haber una mejora para todos hasta que también el racismo fuera enfrentado y destruido. Se trata de una innovación radical e importante. Sitúa el trabajo remunerado de las mujeres como algo indisolublemente atado no solo al sexismo sino también al racismo, elevando el espectro analitico hacia una lógica más amplia y sistémica, aunque sin abordarla completamente. Lamentablemente, su análisis fue rápidamente ignorado. El Partido Comunista de los Estados Unidos y el movimiento sindical

impidieron que muchos consiguieran recursos estatales en el mismo grado que las familias blancas (Nadasen, 2005). 
ante los cuales Jones planteó sus exigencias no supieron aprovechar estas ideas y las feministas mainstream las ignoraron.

Estas últimas sí abordaron la doble carga de trabajo remunerado y no remunerado, pero sin reconocer toda la rica complejidad de la racialización del trabajo de las mujeres. La "Declaración de Propósitos" de la Organización Nacional de Mujeres (NOW, por su sigla en inglés) de 1966, por ejemplo, plantea que la tecnología puede liberar a las mujeres de las tareas domésticas, definidas por las autoras como "una de las partes más importantes" de la vida de las mujeres. Rechazando la idea de que las mujeres deberian elegir entre la maternidad o la carrera, el documento propone matrimonios "colaborativos", centros de cuidado infantil y capacitación laboral, haciéndose eco de las políticas individualistas y basadas en el mercado que proponía el feminismo de la etapa previa. Dichas medidas promueven la independencia económica de las mujeres, ayudando a sacarlas de los empleos mal remunerados del sector servicios. Las autoras de la "Declaración" hacen observaciones sobre la naturaleza racializada de la fuerza de trabajo, pero no realizan análisis ni sacan conclusiones políticas al respecto.

En estos 150 años de pensamiento feminista sobre el trabajo, vemos una reivindicación continua del valor social del trabajo doméstico y una creciente disposición a inspeccionar críticamente las condiciones bajo las cuales las mujeres lo llevan a cabo. Thompson y Wheeler identifican su importancia para la reproducción y expansión del capitalismo en la década de 1820, pero la mayoría de las socialistas posteriores no continúan este incipiente análisis político-económico. En su lugar, lo que hacen es elaborar una crítica moral y promover variantes del feminismo de la igualdad. Del mismo modo, el esfuerzo de Jones para racializar el análisis del trabajo remunerado y no remunerado no encontró eco. Por el contrario, en la década de 1960, la emancipación de las mujeres se define en gran medida en términos de la libertad de elegir entre diferentes tipos de trabajo: maternidad o carrera, o ambos.

Podría decirse que la visión del carácter emancipatorio del trabajo remunerado ha dominado la agenda de feminismo liberal desde entonces. Esta perspectiva fue parte de los análisis e iniciativas de las feministas que, en los años 70 y 80, buscaban integrar a las mujeres de los países del Sur global en la economía formal (Rathgeber, 1990; Reeves y Baden, 2000). Y, a pesar de las críticas, las Naciones Unidas y muchas agencias no gubernamentales continúan trabajando dentro de este paradigma, más recientemente bajo su forma neoliberal que promueve la extensión de microcréditos a las mujeres pobres para financiar sus emprendimientos productivos (Rankin, 2001). El acceso a la educación superior, a trabajos profesionales y a juntas ejecutivas es la estrategia clave del feminismo dominante actualmente en el Norte global. Ya se trate de 
líderes políticas como Hillary Clinton -en cuya cuenta de Twitter se lee: "Para cada niña que sueña a lo grande: Sí, puedes ser lo que quieras, incluso presidenta"- o de líderes empresariales como Sheryl Sandberg, autora de Lean In, el "feminismo corporativo" (Burnham, 2013) retoma hoy este punto de la crítica feminista: el argumento de que la libertad de las mujeres requiere igualar sus oportunidades en un mercado laboral (y ejecutivo corporativo) desigual.

Una perspectiva feminista de estas características no escapa a las contradicciones asociadas con el feminismo de la igualdad de siglos pasados. Por importante que sea para las mujeres ser tratadas igual que los varones en tanto fuerza de trabajo, la gran mayoria de las mujeres, en el Sur y en el Norte global, realmente no tienen la posibilidad de elegir entre la maternidad y su carrera; ni siquiera pueden elegir su empleo. Y la mayoria de ellas trabajan en empleos tan alejados de las salas de juntas ejecutivas de las compañías, que "optar por eso" no tiene ningún significado en sus vidas. Además, saben muy bien que la opresión de las mujeres no se detiene en las puertas de la fábrica o de la oficina. Para aquellas que tienen trabajos bien remunerados, elegir la maternidad regularmente depende de que otras mujeres (y en general otrorizadas, por ejemplo, racializadas) estén disponibles para asumir la pesada carga del trabajo doméstico, el equivalente en el siglo XXI de la "simple sirvienta" de Wollstonecraft, que se hace cargo de "la parte servil de las tareas domésticas".

\section{Una economía política del trabajo de las mujeres}

Esto es lo que hace tan importante e inspirador, hoy en día, el revival de la visión socialista utópica sobre el trabajo doméstico que tuvo lugar en el siglo XX. Esa historia comienza con la publicación en 1969 de "La economía política de la liberación de la mujer" de Margaret Benston. Con este artículo, Benston logra ir más allá de una crítica moral-política del trabajo doméstico como algo aislante y opresivo y desarrolla un análisis socio-materialista del mismo. Al hacerlo, logró sentar las bases para una teoría de la naturaleza intrínsecamente patriarcal del capitalismo. Utilizando conceptos marxistas, planteó que el trabajo doméstico es trabajo productivo desde el punto de vista económico (y, por lo tanto, capitalista). ${ }^{7}$ Es decir, las tareas domésticas que realizan las mujeres para que sus esposos e hijos sean trabajadores en el presente y en el futuro

7. El trabajo "productivo", en términos marxistas, refiere a aquello que genera directamente plusvalía para los capitalistas. Benston y muchas otras feministas de la reproducción social a menudo se confunden entre este significado analítico específico del término y una definición más convencional. 
son esenciales para los procesos capitalistas de creación de valor. ${ }^{8}$ Esto se debe a que los productos del trabajo doméstico -la alimentación, el vestido y el cuidado de los cuerpos- aseguran un suministro continuo de la fuerza de trabajo necesaria para generar valor económico (o de cambio) para el capital. En otras palabras, el trabajo doméstico de las mujeres reproduce socialmente la fuerza de trabajo para el capital. ${ }^{9}$

Sin embargo, al estar organizado en hogares privados y ser llevado a cabo por mujeres que lo realizan no por una paga sino porque las relaciones patriarcales de género así lo exigen, el trabajo doméstico no es algo cuyo valor pueda medirse de forma capitalista. Por lo tanto, se lo considera improductivo (tanto en la economía marxista como en las estimaciones mainstream de la actividad económica de una sociedad, como el Producto Bruto Nacional). ${ }^{10}$ De igual modo, al ser caracterizado como un asunto privado, se le otorga poco reconocimiento cultural. Por lo tanto, mientras el trabajo doméstico se organice de esta manera, argumenta Benston, las mujeres no pueden ser libres: "La igualdad de acceso a los trabajos fuera del hogar, si bien es una de las condiciones previas para la liberación de las mujeres, en sí misma no será suficiente para otorgarles igualdad; mientras el trabajo en el hogar siga siendo una cuestión de producción privada y sea responsabilidad de las mujeres, simplemente llevarán una doble carga de trabajo" (Benston, 1969, p. 21). La comunidad política e intelectual feminista internacional saludó con entusiasmo el artículo de Benston, debatiendo y desarrollando aún más sus premisas (Miles, 1993). Durante la mayor parte de la década, las feministas lidiaron con una cantidad de preguntas. ¿Cuál es la relación entre el trabajo doméstico y el trabajo productivo en la formación de valor? ¿El primero crea directamente plusvalía o no? ¿Cómo se entrecruza efectivamente la organización patriarcal del trabajo doméstico con la organización capitalista del trabajo productivo? Más que un simple debate académico, el "Debate sobre el trabajo doméstico" impulsó la campaña internacional Salarios para el Trabajo Doméstico (IWHW, por su sigla en inglés), lanzada en 1972 (Katsarova, 2015). Las cofundadoras de la campaña, Mariarosa Dalla Costa y Selma James, argumentaron (en contra de Benston, quien sostenía que el trabajo doméstico constituía un modo de producción diferenciado no capitalista) que el trabajo doméstico es trabajo productivo, en el sentido capitalista. Y como es directamente creador de plusvalía, razonaron Dalla Costa y James, las mujeres que lo realizan tienen derecho a un salario.

8. Benston está hablando de una familia nuclear heterosexual.

9. Benston, sin embargo, no usó el término trabajo socialmente reproductivo.

10. El concepto marxista de trabajo productivo fue pensado para revelar la lógica del capital, mientras que la economía convencional lo utiliza para medir la producción. 
Si bien la campaña IWHW efectivamente politizó el trabajo doméstico, sus criticas plantearon diferentes preocupaciones. Algunas señalaron que realizar pagos por las tareas domésticas extendería la explotación capitalista a la esfera doméstica, mientras que otras interpretaron la campaña, y el feminismo de la reproducción social en general, como una universalización de las experiencias de opresión de las mujeres que implicaba, de modo reduccionista, suponer que todas las mujeres comparten la devaluación, el aislamiento y la invisibilidad de las amas de casa de clase media (Weeks, 2011; Molyneux, 1979). Mientras esto ocurria, el feminismo marxista perdía terreno como corriente intelectual. Heidi Hartmann, en su famosa crítica (1979), argumentaba que el feminismo marxista subsumía, política y teóricamente, los problemas de las mujeres en la lucha de clases, y fallaba en analizar el supuesto interés material de los varones en la opresión de las mujeres. A su vez, un feminismo negro revivido señalaba el fracaso del feminismo marxista por su falta de compromiso teórico con los problemas del racismo (Mohanty, 1984; Davis, 1982). Para la década de 1980, una ofensiva agresiva de la clase dominante había derrotado efectivamente a los movimientos obreros y sociales que antes habian galvanizado las disidencias, y el proyecto de articular una teoria integral del capitalismo patriarcal basado en una crítica política económica del trabajo reproductivo de las mujeres perdió impulso.

Aunque la mayoria de las feministas de izquierda cambiaron a Marx por Derrida y Foucault, hubo quienes continuaron desarrollando el paradigma del feminismo de la reproducción social (ver Bezanson y Luxton, 2006). Un avance importante se produjo con la publicación de Marxism and the Oppression of Women: Toward a Unitary Theory, en 1983. Su autora, Lise Vogel, retoma el debate sobre el trabajo doméstico, pero va un paso más allá de sus impases políticos y teóricos. Ella argumenta (con Benston y contra Dalla Costa y James) que el trabajo doméstico produce valores de uso (no valor de cambio). Y además plantea (contra Benston y con Dalla Costa y James) que el trabajo doméstico no constituye un modo de producción fuera del capitalismo. Específicamente, Vogel avanza en la comprensión del capitalismo como una dinámica económica y extraeconómica entrelazada y contradictoria. En su lectura, el patriarcado y el capitalismo no son dos sistemas distintos que se cruzan, sino que forman un sistema inseparable, unitario, en el que ninguna forma de dominación es reductible a la otra. Como ya he planteado en otra parte, Vogel traslada el análisis del capitalismo a un terreno más dialéctico, uno que posiciona sus partes (por ejemplo, las relaciones trabajo / capital y las relaciones de género) como aspectos integralmente vinculados de una totalidad capitalista, que sostiene y reproduce la diferencia o la particularidad dentro de esa totalidad (Ferguson, 2016). Todo esto 
lo logra basándose y extendiendo una teoría de la dualidad del trabajo de reproducción social, insistiendo en que el trabajo reproductivo es, al mismo tiempo, concreto y abstracto, está a la vez dentro y fuera de la relación directa capital/trabajo, y es al mismo tiempo contradictorio con el capitalismo y esencial para su reproducción.

Es preciso hacer una aclaración antes de abandonar la discusión sobre la contribución de Vogel. Puede parecer que ella simplemente está reafirmando la preocupación de la clase media blanca por las tareas domésticas de las mujeres porque el enfoque todavía está puesto en el trabajo doméstico no remunerado, pero no es así. El avance teórico clave de Vogel es ubicar la opresión de las mujeres no en el trabajo doméstico per se, sino en las relaciones generizadas de la reproducción biológica y social de las personas, y específicamente en las contradicciones que caracterizan la relación de la reproducción social con el trabajo asalariado. Debido a que las mujeres, en virtud de la organización social de sus capacidades biológicas para dar a luz y amamantar, están situadas en el meollo de esa contradicción, Vogel postula que esta relación proporciona una lógica socio-material a la opresión continua que experimentan las mujeres bajo el capitalismo. Esto no es lo mismo que decir que las experiencias de todas las mujeres como trabajadoras domésticas son inherentemente opresivas. Más bien, el punto es que la incesante y poderosa necesidad de trabajadores que tiene el capitalismo -para la (re) producción continua de la vida- lo lleva a incorporar a todas y a cada una de las prácticas, instituciones y relaciones en la persecución de ese fin. La familia privatizada y el trabajo doméstico de las mujeres ha sido, y sigue siendo, un medio preferencial para producir fuerza de trabajo. Pero no es el único (piénsese en el papel que juegan al respecto las escuelas, los hospitales, las guarderias, los restaurantes y las cárceles). ${ }^{11}$ Tampoco la familia está siempre organizada según líneas patriarcales: las madres solteras y jefas de familia siguen oprimidas, por ejemplo, pese a que su trabajo doméstico no beneficia a ningún varón adulto en particular.

\section{La complejidad del trabajo de reproducción social en el capitalismo}

Otra forma de pensar este problema es considerar que los cuerpos de los trabajadores no son accidentales en relación al proceso capitalista de acumulación. Deben ser disciplinados y diferenciados, para ser trabajadores, pero también para ser diferentes tipos de trabajadores. Algunos - muchos- deben ser trabajadores dispuestos (o que no tienen

11. Para ciertas discusiones sobre el papel del estado en la reproducción social véase Picchio (1992) y Seccombe (1993). 
más remedio) a realizar trabajos peligrosos y arduos a horas irregulares por un salario bajo. En otras palabras, deben ser trabajadores que puedan reproducirse a sí mismos y a los demás de manera que sus costos de reproducción social sean lo más bajos posibles. Desde el punto de vista del capitalismo a nivel mundial, se observa que los cuerpos, y su inserción en los procesos de reproducción social, no solo tienen diferencias biológicas. También están ubicados espacial y socialmente en un terreno capitalista global marcado por las fronteras nacionales y las relaciones imperialistas de poder (ver Ferguson y McNally, 2014). Estas relaciones geo-sociales jerárquicas crean oportunidades para el abaratamiento sistémico de algunas vidas, un proceso que aprovecha y refuerza las prácticas e instituciones racistas, colonialistas (así como sexistas y heterosexistas). Esta dinámica sale a la luz cuando se examina la reproducción social de la fuerza de trabajo a través de la diferencia socio-espacial, al rastrear, por ejemplo, los regímenes de migración dentro de las naciones o a través de las fronteras internacionales. Dichos regimenes pueden encontrarse a lo largo de la larga historia del capitalismo, desde la gran ola de trabajadores irlandeses que consiguieron empleos en las fábricas textiles, en los altos hornos y en hogares burgueses de Gran Bretaña en el siglo XIX, pasando por los millones de africanos esclavizados que trabajaron en las plantaciones estadounidenses, hasta llegar a nuestros dias con los más de 200 millones de trabajadores migrantes internacionales que se ocupan de los hijos de otras personas, levantan las cosechas, procesan productos agrícolas y extraen las riquezas de la tierra en países distintos de aquellos en los que tienen ciudadanía.

De ningún modo se trata simplemente de un movimiento "libre" de mano de obra "libre": la llegada a las puertas del capital de una fuerza laboral relativamente barata, ya racializada y/o colonizada, es el resultado de fuerzas de dominación y coerción. El carácter coercitivo del mercado de trabajo global capitalista implica, en primera instancia, el despojo de comunidades indígenas, pequeños propietarios de tierras y trabajadores artesanales avalado por el estado, y el debilitamiento de las unidades de producción doméstica (Mies, 1998; Federici, 2004). También implica la supervivencia de diversas formas de trabajo no libre, desde la esclavitud absoluta hasta la servidumbre por deudas y los llamados migrantes temporales a los que se les niegan los derechos básicos de ciudadanía (LeBaron, 2014; Banaji, 2003). ${ }^{12}$ Se ejerce en un contexto global de guerras, fronteras, acuerdos comerciales y de inversión, y vigilancia policiaca, mecanismos que aseguran a los capitalistas no

12. Banaji sugiere que, históricamente, el trabajo capitalista se caracteriza por diversos grados de falta de libertad. 
cualquier tipo de fuerza de trabajo, sino trabajadores que son sujetos coloniales y racializados. Y como la mano de obra es cada vez más barata de reproducir, se vuelve, de hecho, "desechable" (Wright, 2006). Es decir, hay un punto en el que los trabajadores se reproducen socialmente a un costo tan bajo que la reproducción simultánea de la vida humana y de la fuerza de trabajo ya no es, en gran medida, una contradicción. Pues, en este punto, Alessandra Mezzadri sugiere: "El capital puede consumir los cuerpos de los trabajadores sin mayores preocupaciones por la rentabilidad. Después de todo, cuando unos se agotan hay otros disponibles a los mismos precios baratos" (2017, p. 161).

Sin embargo, para comprender más plenamente la deshumanización diferencial de los cuerpos laborales que se produce como parte del mantenimiento de las relaciones sociales capitalistas, debemos prestar atención no solo al marxismo y al feminismo de la reproducción social, sino también a las tradiciones feministas centradas en problemas de diferencia, cultura e identidad.

Robin Truth Goodman realiza justamente este trabajo en su libro de 2013, Gender Work: Feminism after Neoliberalism, trazando una linea de pensamiento sobre el "trabajo de las mujeres" que revisa ciertos postulados posestructuralistas sobre el tema. Plantea que el "trabajo de las mujeres" (el trabajo de cuidado, servicio, afecto y socialización) desempeña el papel del lenguaje en la organización de lo social (más precisamente en la explicación de Aihwa Ong sobre la lógica cultural del capitalismo global). Sin embargo, estos poderes significantes del trabajo de las mujeres se rompen justamente porque, como ha remarcado Kristeva, la vida excede la representación. Los sujetos se forman, por ende, en relación tanto con el trabajo como con "un imaginario no conceptual, donde somos más que lo que hacemos dentro de la matriz productiva global y sus entidades" (Goodman, 2013, p. 108). Goodman relaciona esta preocupación culturalista por la formación del sujeto con la contradicción entre producción y reproducción propia del capitalismo, siguiendo la crítica de Michael Hardt y Antonio Negri sobre la tendencia del capitalismo neoliberal a subsumir la vida en el trabajo. En esta línea, enfatiza que la "llamada feminización del trabajo" que identifican Hardt y Negri es el mecanismo del neoliberalismo para intensificar la explotación (Goodman, 2013, p. 171). No es cualquier sujeto del trabajo el que tiene valor para el capital; es el sujeto trabajador devaluado por su feminización previa y continua. Melissa Wright ofrece un ejemplo convincente de esta tendencia en su estudio sobre las trabajadoras de una fábrica de televisión mexicana. En su libro Disposable Women and other Myths of Global Capitalism, se basa en la teoria de la subjetividad encarnada de Butler para mostrar cómo las mexicanas feminizadas son valoradas como trabajadoras de las maquiladoras precisamente porque 
su (mítica) "corporalidad del tercer mundo y femenina" (2006, p. 14) las marca como "perpetuamente descalificadas e inexpertas" (2006, p. 47): cuerpos que se utilizarán y eliminarán cuando se agote su valor para el capital.

Pero, observa Goodman, aunque el "trabajo de las mujeres" es interno al capitalismo, permanece separado de él. Esto se debe a que la reproducción de la vida -producir para satisfacer las necesidades humanas que sostendrán la fuerza de trabajo presente y futura- no es reducible al ritmo y el pulso de la producción capitalista. Exige una temporalidad diferente. El trabajo de reproducción biológica, cuidado, servicio y socialización que está involucrado en la producción de sujetos que trabajan, crea y satisface necesidades y deseos que son "excesivos para el capital, incontenibles o intraducibles" (2006, p. 160). Como tal, el "trabajo de las mujeres" constituye las bases creativas para ir más allá del capitalismo. ${ }^{13}$ Rosemary Hennessy aborda esta misma cuestión acerca de la interacción contradictoria entre el valor cultural y valor económico en la producción de sujetos de trabajo al entablar debates en la teoría queer sobre "la materialidad del afecto". Aunque acepta que los afectos (las sensaciones y emociones que se registran en el cuerpo) son múltiples e indefinidos, también plantea que su "indeterminación está basada en la organización histórica de las relaciones sociales que sostienen la vida" (2013, p. 44) o, más precisamente, que producen y satisfacen las necesidades humanas. Dada la presión sistémica para reducir los costos de los salarios y los servicios sociales de los que depende la supervivencia de la clase trabajadora para aumentar la rentabilidad, la "organización histórica" capitalista se caracteriza por la producción sistémica de necesidades humanas insatisfechas. Hennessy argumenta que el trabajo reproductivo gestiona constantemente una "cultura afectiva" dominante que exige disciplinar sensaciones y sentimientos expansivos para crear valor para el capital (2013, p. 50). Además, debido a que los trabajadores venden parte de sí mismos (sus capacidades humanas) al capital por un salario, todos usan lo que ella llama "una segunda piel": una identidad encarnada (degradada) que parece natural pero que de hecho es cambiante, "está abierta a la historia... [y de este modo se convierte] en un espacio de lucha" (2013, p. 126). Y aquellos trabajadores que atienden las necesidades más íntimas de las personas para el cuidado corporal y emocional (el trabajo remunerado y no remunerado de alimentar y cuidar de niños y ancianos y las tareas domésticas) tienen segundas pieles particularmente feminizadas, sexualizadas y / o racializadas. 


\section{Conclusión}

Goodman, Wright y Hennessy extienden el análisis de la reproducción social para dar cuenta de la particularidad del sujeto trabajador. Conscientes de la dinámica cultural y los procesos encarnados de la formación de sujetos, sus contribuciones lidian con las complejidades relacionadas con la teorización del trabajo como una realidad capitalista concreta y vivida. Este es un concepto de trabajo que identifica condiciones capitalistas de despojo y acumulación que son comunes a todos los sujetos trabajadores, así como también lo es la relación contradictoria entre producción y reproducción capitalista; pero, al mismo tiempo, reconoce que el trabajo se diferencia en y a través de las relaciones racializadas, sexualizadas y de género que inciden en esas condiciones. A su vez, es un concepto de trabajo que pone en primer plano el aspecto generativo y creativo de la actividad humana práctica cotidiana de las personas, actividad que contiene el potencial de crear nuevos mundos de nuevas maneras. Por lo tanto, un enfoque feminista de la reproducción social invoca y se nutre de un rico terreno teórico, que va mucho más allá de la evaluación optimista del feminismo de la igualdad sobre el trabajo remunerado como ámbito de la igualdad y la libertad de las mujeres, y también de las contradicciones asociadas con esa posición. Sugiere que es la organización capitalista del trabajo, remunerado y no remunerado, lo que perpetúa la falta de libertad de género (como también lo referido a la racialización, la heterosexualización y la colonización), viendo en el trabajo de reproducción social el impedimento sistémico para satisfacer las necesidades de la vida. Y aconseja el rechazo a esa organización y una reconfiguración de las relaciones laborales de manera que se alineen y satisfagan con las necesidades humanas.

\section{Referencias}

Banaji, J. (2003). The Fictions of Free Labour: Contract, Coercion and So-called Unfree Labour. Historical Materialism, 11 (3), 69-95.

Benston, M. (1969). The Political Economy of Women's Liberation. Monthly Review, 21 (4), 13-27.

Bezanson, K. y Luxton, M. (2006). Introduction: Social Reproduction and Feminist Political Economy. En K. Bezanson y M. Luxton (eds.), Social Reproduction: Feminist Political Economy Challenges Neo-Liberalism (pp. 3-11). McGill-Queen's UP.

Brown Blackwell, A. (1975). Relation of Women's Work in the Household to Work Outside. En A.S. Kraditor, Up from the Pedestal: Selected Writings in the History of American Feminism (pp. 159-159). Quadrangle Books. Burnham, L. (2013) 1\% Feminism. Open Democracy, 13 de abril. https:// 
www.opendemocracy. net/5050/linda-burnham/1-feminism. Consultado el 1 de agosto de 2017.

Clark, A. (1992). Working Life of Women in the Seventeenth Century. Routledge [1919].

Clinton, H. (2016). "To Every Little Girl Who Dreams Big: Yes, You Can Be Anything You Want. Even President. Tonight Is for You. H.” Twitter, 7 de junio, 6:08 p.m. https://twitter.com/HillaryClinton/status / 740349871073398785.

Dalla Costa, M. y Jones, S. (1973). The Power of Women and the Subversion of the Community. Falling Wall Press.

Davis, A. (1982). Women, Race and Class. Women's Press.

De Gouges, O (2017). Declaration of the Rights of Women and Citizens [1791]. [Trad. esp.: O. De Gouges et al. (2007), Cuatro mujeres en la Revolución Francesa. Biblos]

Engels, F. (1972). The Origin of the Family, Private Property and the State. International [1884].

Federici, S. (2004). Caliban and the Witch: Women, the Body and Primitive Accumulation. Autonomedia.

Ferguson, S. (2016). Intersectionality and Social-Reproduction Feminisms: Toward an Integrative Theory. Historical Materialism, 24 (2), 38-60.

Ferguson, S. y McNally, D. (2014). Precarious Migrants: Gender, Race and the Social Reproduction of a Global Working Class. En L. Panitch y G. Albo (eds.). Socialist Register 2015: Transforming Classes (pp. 1-23). Merlin Press.

Goodman, R. T. (2013). Gender Work: Feminism after Neoliberalism. Palgrave Macmillan.

Hartmann, H. (1979). The Unhappy Marriage of Marxism and Feminism: Towards a More Progressive Union. Capital \& Class, 3 (2), 1-33.

Hayden, D. (1982). The Grand Domestic Revolution: A History of Feminist Designs for American Homes, Neighborhoods, and Cities. MIT Press.

Hennessy, R. (2013). Fires on the Border: The Passionate Politics of Labor Organizing on the Mexican Frontera. University of Minnesota Press.

Jones, C. (1949). An End to the Neglect of the Problems of the Negro Woman! Political Affairs. National Women's Commission, CPUSA. University of California Digital Library, junio, p. 5. http://purl.flvc.org/FCLA/ DT/1927554. Consultado el 1 de agosto de 2017.

Katsarova, R. (2015). Repression and Resistance on the Terrain of Social Reproduction: Historical Trajectories, Contemporary Openings. Viewpoint Magazine, 5, 31 de octubre. https://www.viewpointmag. com/2015/10/31/repression-and-resistance-on-the-terrain-of- social-reproduction-historical-trajectories-contemporary-openings/. Consultado el 1 de agosto de 2017.

LeBaron, G. (2014). Unfree Labor beyond Binaries: Social Hierarchy, Insecurity, and Labor Market Restructuring. International Feminist Journal of Politics, 17 (1), 1-19. 
Mezzadri, A. (2017). The Sweatshop Regime: Labouring Bodies, Exploitation, and Garments Made in India. Cambridge University Press.

Mies, M. (1998). Patriarchy \& Accumulation on a World Scale: Women in the International Division of Labour. Zed Books.

Miles, A. (1993). Margaret Benston's Political Economy of Women's Liberation: International Impact. Canadian Women's Studies/Les cahiers de la femme, 13(2), 31-35.

Mohanty, C.T. (1984). Under Western Eyes: Feminist Scholarship and Colonial Difference. Boundary, 2 (12-13), 333-358.

Molyneux, M. (1979). Beyond the Domestic Labor Debate. New Left Review, 116, 3-27.

Nadasen, P. (2005). Welfare Warriors: The Welfare Rights Movement in the United States. Routledge.

National Organization for Women (1966). http://now.org/about/history/ statement-of-purpose/. Consultado el 1 de agosto de 2017.

Perkins Gillman, C. (1975). Economic Basis of the Woman Question. En A.S. Kraditor (ed.) Up from the Pedestal: Selected Writings in the History of American Feminism (pp. 175-178). Quadrangle Books [1898].

Picchio, A. (1992). Social Reproduction: The Political Economy of the Labour Market. Cambridge University Press.

Pinchbeck, I. (1985). Women Workers and the Industrial Revolution 17501850. Virago Press [1930].

Radcliffe, M.A. (1799). The Female Advocate: Or an Attempt to Recover the Rights of Women from Male Usurpation. Vernor and Hood. http://ota. ox.ac.uk/text/5092.html. Consultado el 1 de agosto de 2017.

Rankin, K.N. (2001). Governing Development: Neoliberalism, Microcredit, and Rational Economic Woman. Economy and Society, 30 (1), 18-37.

Rathgeber, E. M. (1990). WID, WAD, GAD: Trends in Research and Practice. The Journal of Developing Areas, 24 (4), 289-302.

Reeves, H. y Baden, S. (2000). Gender and Development: Concepts and Definitions. Institute of Development Studies.

Seccombe, W. (1993). Weathering the Storm: Working-Class Families from the Industrial Revolution to the Fertility Decline. Verso Books.

Siegel, R. B. (1993-1994). Home as Work: The First Women's Rights Claims Concerning Wives' Household Labor, 1850-1880. Yale Law Journal, 103, 1073-1217.

Stern, E. (1975). Women Are Household Slaves. En A.S. Kraditor, Up from the Pedestal: Selected Writings in the History of American Feminism (pp. 346-353). Quadrangle Books.

Thompson, W. (1825). Appeal of One Half the Human Race, Women, Against the Pretensions of the Other Half, Men, to Retain Them in Political, and Thence in Civil and Domestic Slavery. Longman, Hurst Rees, Orme, Brown \& Green.

Weeks, K. (2011). The Problem with Work: Feminism, Marxism, Antiwork Politics, and Postwork Imaginaries. Duke UP. 
Wollstonecraft, M. (1999). Vindication of the Rights of Women [1792]. Bartleby. http://www.bartleby.com/144/. Consultado el 1 de agosto de 2017. Wright, M. (2006). Disposable Women and Other Myths of Global Capitalism. Routledge. 